\title{
PRONTOX - proton therapy to reduce acute normal tissue toxicity in locally advanced non-small-cell lung carcinomas (NSCLC): study protocol for a randomised controlled trial
}

\author{
Sebastian Zschaeck ${ }^{1 *}$, Monique Simon ${ }^{2}$, Steffen Löck ${ }^{3}$, Esther G. C. Troost ${ }^{4}$, Kristin Stützer ${ }^{5}$, Patrick Wohlfahrt ${ }^{5}$, \\ Steffen Appold ${ }^{6}$, Sebastian Makocki ${ }^{7}$, Rebecca Bütof ${ }^{7}$, Christian Richter ${ }^{8}$, Michael Baumann ${ }^{9}$ \\ and Mechthild Krause ${ }^{10}$
}

\begin{abstract}
Background: Primary radiochemotherapy with photons is the standard treatment for locally advanced-stage non-small cell lung cancer (NSCLC) patients. Acute radiation-induced side effects such as oesophagitis and radiation pneumonitis limit patients' quality of life, and the latter can be potentially life-threatening. Due to its distinct physical characteristics, proton therapy enables better sparing of normal tissues, which is supposed to translate into a reduction of radiation-induced side effects.

Methods/design: This is a single-centre, prospective, randomised controlled, phase II clinical trial to compare photon to proton radiotherapy up to 66 Gy (RBE) with concomitant standard chemotherapy in patients with locally advanced-stage NSCLC. Patients will be allocated in a 1:1 ratio to photon or proton therapy, and treatment will be delivered slightly accelerated with six fractions of 2 Gy (RBE) per week.

Discussion: The overall aim of the study is to show a decrease of early and intermediate radiation-induced toxicity using proton therapy. For the primary endpoint of the study we postulate a decrease of radiation-induced side effects (oesophagitis and pneumonitis grade II or higher) from 39 to 12\%. Secondary endpoints are locoregional and distant failure, overall survival and late side effects.
\end{abstract}

Trial registration: Registered at ClinicalTrials.gov with Identifier NCT02731001 on 1 April 2016.

Keywords: Proton radiotherapy, Non-small-cell lung cancer (NSCLC), Locally advanced, Photon radiotherapy, Toxicity, Randomised clinical trial, Phase II trial

\footnotetext{
* Correspondence: sebastian.zschaeck@uniklinikum-dresden.de 'Department of Radiation Oncology, Faculty of Medicine and University Hospital Carl Gustav Carus, Technische Universität Dresden, OncoRay National Center for Radiation Research in Oncology, Faculty of Medicine and University Hospital Carl Gustav Carus, Technische Universität Dresden and Helmholtz-Zentrum Dresden - Rossendorf, German Cancer Consortium (DKTK) Dresden and German Cancer Research Center (DKFZ), National Center for Tumor Diseases (NCT), Partner Site Dresden, Fetscherstr. 74, Dresden 01307, Germany

Full list of author information is available at the end of the article
} 


\section{Background}

Primary lung tumours are a leading cause for tumourrelated mortality worldwide, which is mainly due to their unfavourable prognosis, particularly in the advanced or metastasised stages. Non-small cell lung cancer (NSCLC) accounts for the vast majority of primary lung cancers. The treatment of choice for inoperable or locally advanced NSCLC without distant metastasis is primary radiochemotherapy [1]. The prescribed standard radiation dose ranges between 60 and 66 Gy in 2-Gy fractions, delivered using three-dimensional conformal or intensity-modulated radiation therapy (3D-CRT and IMRT, respectively). As local failure occurs in about $50 \%$ of patients with locally advanced disease, current approaches aim for escalation of radiation dose. The phase III RTOG 0617 trial randomised patients to 60 Gy standard dose or 74 Gy dose escalation after promising phase I and II trials [2]. Unexpectedly, median overall survival of patients in the high-dose group was decreased compared to standard fractionation. The underlying reasons are currently being assessed and may include insufficient target coverage and increased dose to the heart.

In general, pulmonary, oesophageal and cardiac toxicity [3] constitute the most relevant acute and late toxicities after thoracic radiotherapy. Therefore, future studies on improved radiation treatment for NSCLC have to include vigorous reduction of normal tissue complication probabilities. One promising approach is the use of proton beam radiotherapy. Proton therapy offers advantages in normal tissue sparing due to the sharp dose fall-off behind the target volume covered by the spread-out Bragg peak that it produces. In a retrospective analysis, patients with advanced stages of NSCLC treated with proton beam therapy presented with fewer radiation-induced side effects although they were treated with 74 Gy (RBE) (= photon equivalent dose by considering an average relative biological effectiveness (RBE) of 1.1) compared to the photon control group which had a prescribed dose of only 63 Gy [4]. Proton therapy offers unique opportunities for dose escalation in NSCLC due to its sparing of low and intermediate radiation doses to surrounding normal tissues. Before applying dose escalation with proton beam therapy, prospective studies confirming lower normal tissue toxicity for proton therapy should be performed to ensure maximum patient safety.

The aims of the present study are to demonstrate a reduction of acute radiation-induced side effects, i.e. pneumonitis and oesophagitis grade II or higher, using proton versus photon radiochemotherapy to equal radiation doses. Secondary endpoints include evaluation of quality of life, locoregional control, occurrence of distant metastases, survival and late radiation-induced side effects.

\section{Methods/design}

This is a single-centre, prospective, randomised controlled, phase II clinical trial comparing slightly accelerated radiochemotherapy with photons to that with protons in patients with inoperable or locally advanced, cytologically or histologically confirmed NSCLC. Dose prescription to the tumour is $66 \mathrm{~Gy}$ (RBE), maximal doses to the organs at risk comply with international standards. All patients undergo complete clinical staging with Fluorodeoxyglucose-Positron Emission TomographyComputer Tomography (FDG-PET-CT) and magnetic resonance imaging (MRI) of the brain.

\section{Inclusion criteria}

- NSCLC (confirmed by cytology or histology) staged UICC IIIA or IIIB or UICC II if the tumour is medically inoperable or the patient declines surgery

- No distant metastases (M1)

- Patient is aged between 18 and 70 years

- Patient is medically suited for primary radiochemotherapy with curative intent

- Patient has signed a declaration of informed consent

- Adequate compliance for treatment and clinical follow-up

- Adequate contraception during and after therapy if indicated

\section{Exclusion criteria}

- Participation in another interventional trial at the same time

- T1 or T2 N0 tumours that are suitable for stereotactic radiotherapy

- Relevant neurological or psychiatric disorders that hinder treatment, follow-up or understanding of the procedures

- Pregnant or breastfeeding women

- Prior thoracic radiotherapy

- History of other malignancies during the last 5 years (exceptions can be made for tumours with excellent outcome)

- Unintended weight loss greater than 15\% before therapy

- Serological alterations (liver, kidney) prohibiting application of simultaneous chemotherapy

- Respiratory motion of the tumour of more than $10 \mathrm{~mm}$ (evaluated by four-dimensional computer tomography (4D-CT), also when methods for motion reduction (abdominal compression) are applied

\section{Recruitment, randomisation and workflow}

To assess whether patients can be treated with protons, a $4 \mathrm{D}-\mathrm{CT}$ will be performed to evaluate the respiratory motion of the tumour. If tumour motion is larger than $10 \mathrm{~mm}$, abdominal compression or gating methods can be used and the patient will undergo a second 4D-CT 
scan. If the respiratory motion is still too large, the patient will be treated with photons within an observational cohort. For patients with tumour motion of less than $10 \mathrm{~mm}$, the gross tumour volume (GTV), clinical target volume (CTV) and organs at risk (OAR) will be delineated. Patients are then randomised for proton or photon therapy and the respective radiation plans will be calculated. If target coverage is insufficient or doses at OAR are too high, a new treatment plan using the other modality (photons or protons respectively) will be calculated. If the other plan adheres better to the constraints, the patient is treated with the respective modality within an observational cohort. Patient reallocation to another treatment arm due to nonconformity to the constraints will be statistically handled as an event in the randomised (intent-to-treat) arm. This procedure ensures maximum patient safety while not compromising results for randomised patients. Figure 1 shows a flowchart of patient allocation to treatment arms.

\section{Radiotherapy}

Treatment is planned using FDG-PET-CT in the treatment position and an additional 4D-CT to assess tumour motion. On the basis of the 4D-CT an internal gross tumour volume (iGTV) is generated that encompasses the whole tumour motion during respiration. The CTV is generated by summing the iGTVs. Patients within the photon arm will be treated using IMRT with six fractions per week to a total dose of 66 Gy. Patients within the proton arm will receive 66 Gy (RBE) also delivered with six fractions per week. Target coverage and dose to organs at risk will be assessed by at least one physician and one physicist. Constraints will be according to current clinical guidelines in combination with the RTOG 1308 constraints, whichever is stricter than the QUANTEC criteria [5]. During the course of radiotherapy in-room control 4D-CTs (maximum two per week) will be acquired to check for stability of inner anatomy and motion characteristics.

Chemotherapy will be delivered according to current clinical standards without differences for both groups. Figure 2 shows the planned workflow for both treatment arms.

\section{Primary and secondary endpoints}

The primary aim of the study is to show whether a reduction of acute and intermediate (i.e. late-occurring pneumonitis) radiation-induced side effects (pneumonitis and dysphagia grade II or higher scored by Common Terminology Criteria for Adverse Events CTC-AE 4.0) can be achieved using proton as compared to photon beam therapy. The primary endpoint of the study will be the occurrence of radiation-induced side effects (CTC-AE 4.0 scoring) up to 6 months after treatment or re-planning with another radiation modality due to infringement of OAR or tumour coverage constraints. Secondary endpoints contain classification of severity of early and late side effects in both groups, comparison of quality of life

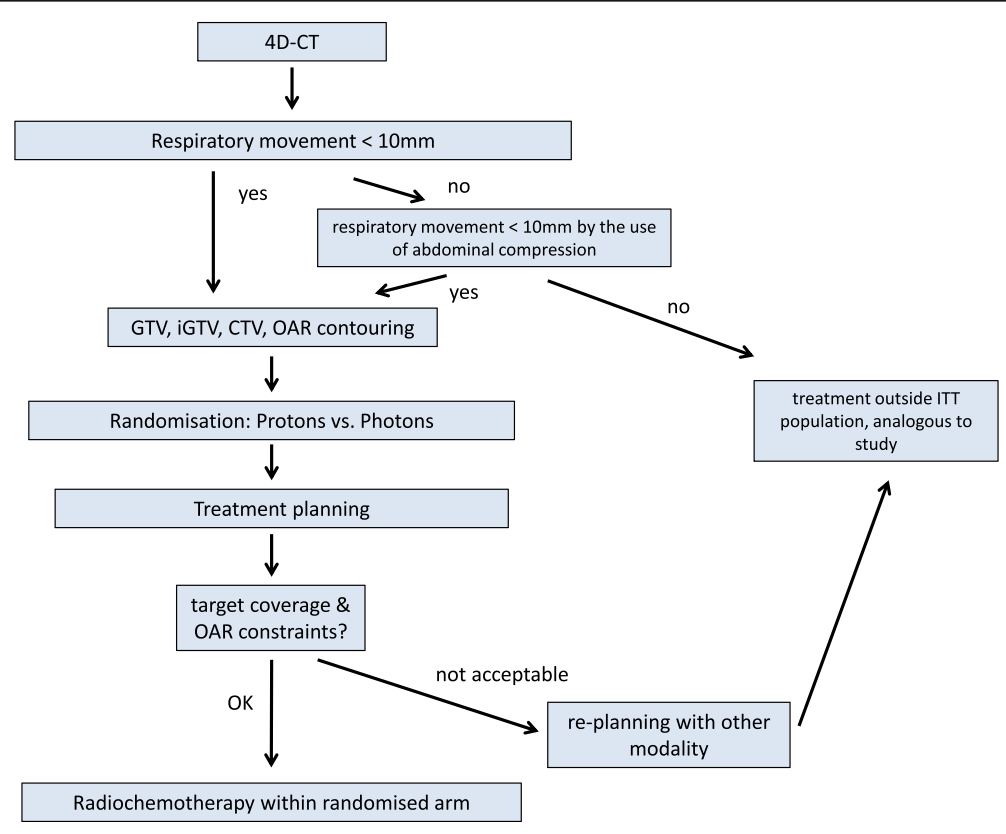

Fig. 1 Flowchart of planning procedures and patient allocation 


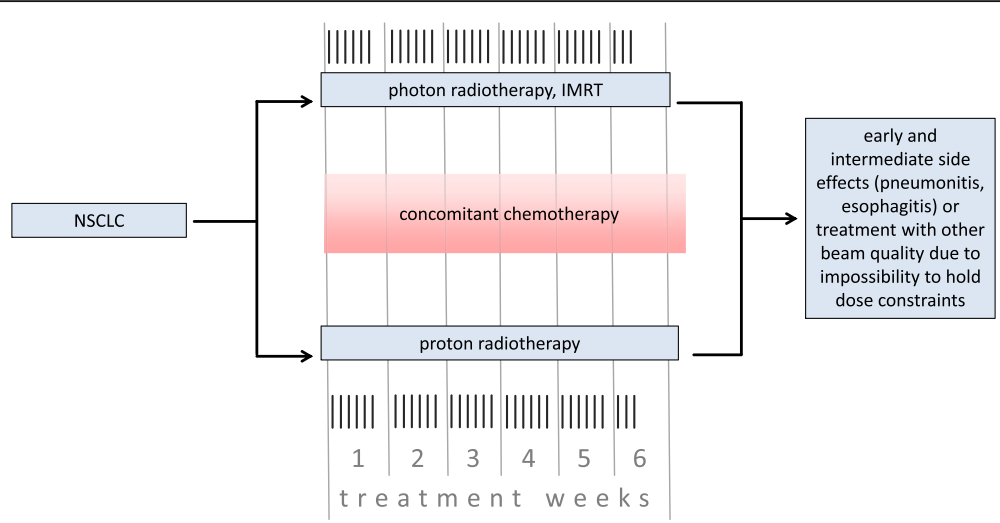

Fig. 2 Flowchart of both randomised treatment arms and primary endpoint

and of treatment outcome; furthermore, dosimetric parameters and $\mathrm{CT}$ alterations of normal tissues at follow-up will be compared between both groups. Quality of life will be assessed additionally by the European Organisation for Research and Treatment of Cancer (EORTC) questionnaires $\mathrm{C} 30$ and $\mathrm{LC} 13$ before and at the end of treatment and at each follow-up visit. For follow-up, patients will be interviewed via telephone $2,4,8$ and 10 weeks after treatment based on a standardised form. Clinical follow-up examinations with additional CT or FDG-PET-CT scans are scheduled 6 weeks, 3 months and 6 months after treatment. After that, follow-up will be continued outside the trial. During therapy and at follow-up, scoring of side effects will be performed according to CTC-AE 4.0. After that, follow-up will be continued outside the trial and scoring of side effects will also be performed according to CTC-AE 4.0.

The trial design and protocol adhere to Standard Protocol items: Recommendations for Interventional Trials (SPIRIT) criteria (www.spirit-statement.org); the SPIRIT checklist and figure can be found as Additional file 1: Table S1 and Additional file 2: Figure S1.

\section{Statistics}

The primary endpoint of the study is the occurrence of acute and intermediate radiation-induced side effects, which will be observed in randomised patient cohorts treated with photon or proton therapy in a 1:1 ratio. For photon therapy, the incidence of pneumonitis and dysphagia grade II or higher at our institution is approximately $18 \%$ and $25 \%$, respectively, which compares favourably with incidences in the published literature $[6,7]$. Using proton therapy, a reduction by a factor of 4-5 seems achievable [5]. Therefore, conservative estimates for pneumonitis and dysphagia occurrence are $4 \%$ and $8 \%$, respectively. Assuming that the two side effects are independent, the combined fraction of expected side effects is $0.18+0.25-0.18 \times 0.25=0.39(39 \%)$ for photon therapy and $0.04+0.08-0.04 \times 0.08=0.12(12 \%)$ for proton therapy.

Using these values, the required patient number to reveal a significant difference in the occurrence of side effects between the two arms is calculated as 39. This number is based on a one-sided test of proportions with a continuity correction using a normal approximation (STATA 11.2, StataCorp LP, College Station, TX, USA, function sampsi). The significance level was set to 0.05 and the power to 0.8 . A one-sided test seems appropriate since a reduced occurrence of side effects is expected from proton treatment. Assuming a 20\% patient dropout the final number of patients per treatment arm is 49 .

\section{Discussion}

The aim of this prospective randomised study is to evaluate whether radiochemotherapy for advanced-stage NSCLC patients with protons leads to decreased radiation-induced side effects as compared to photons. Acute side effects are of particular importance in thoracic tumour sites as compared to other radiation sites. Acute radiation toxicity is expected to decrease quality of life of cancer patients, though mostly only temporarily, and thus is currently being subordinated to potential tumour control in a curative setting. Radiationinduced pneumonitis, however, is a potentially lethal toxicity and can develop into chronic fibrosis, often causing deteriorating in patients' long-time quality of life [8]. Exposure of the lung, and probably also the heart, to low and intermediate radiation doses is highly relevant for the development of pneumonitis and can be reduced by proton therapy (see Fig. 3 for dose distributions for IMRT and proton radiotherapy) [7]. Although there is a strong correlation between radiation dose and local control in preclinical studies and 


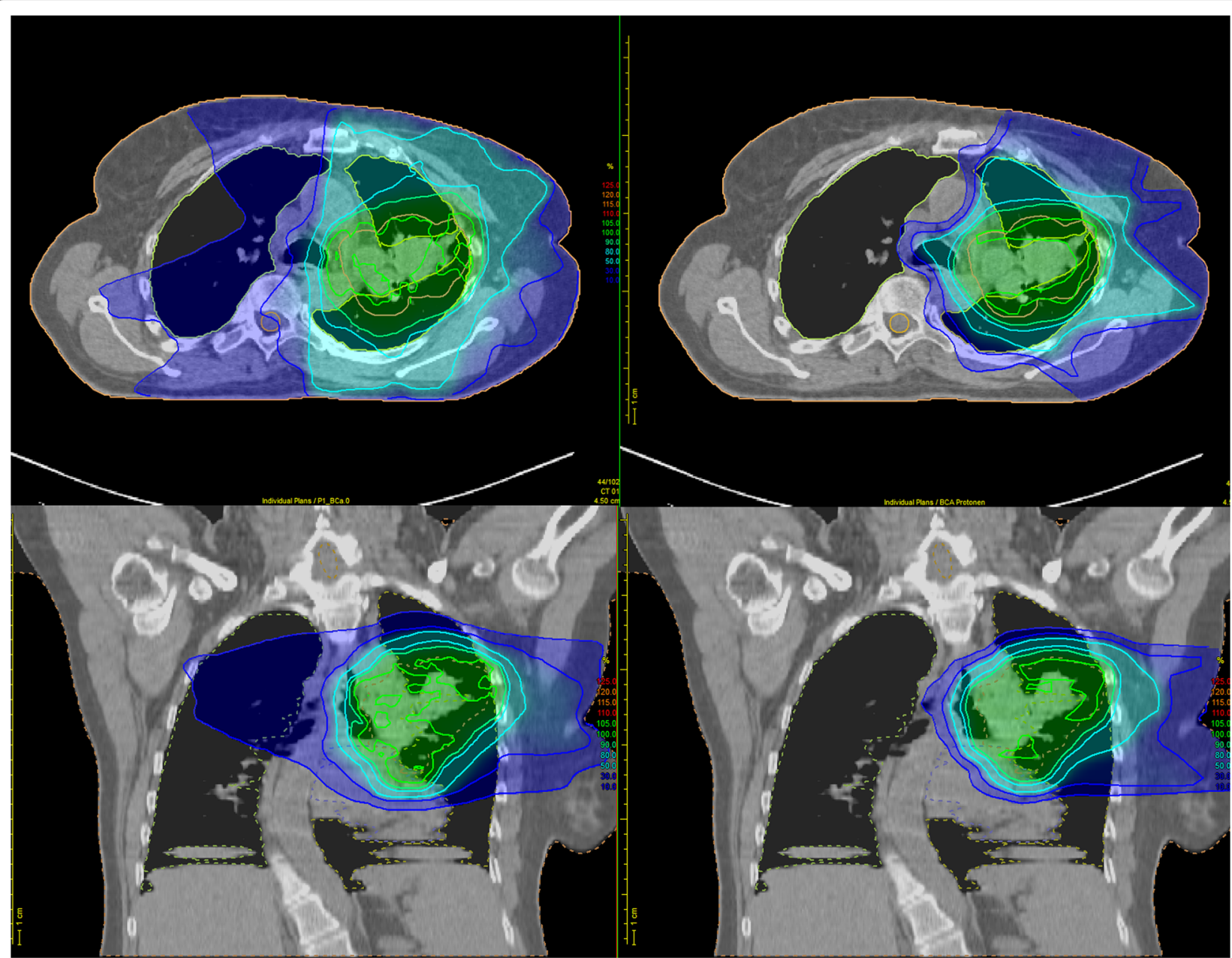

Fig. 3 Dose distribution of one patient with locally advanced non-small cell lung cancer (NSCLC) planned with intensity-modulated radiation therapy (IMRT) (left) or protons (right) showing lower doses to organs at risk (OAR) by proton therapy

in studies using hypofractionated stereotactic radiotherapy or alternative treatment modification, in advanced disease dose escalation with photons did not translate into a survival benefit in the recently published RTOG 0617 study [2, 9-13]. It is noteworthy that in the latter study the dose escalation was performed without acceleration, even though a time factor exists for locally advanced NSCLC as confirmed by the CHARTWEL study [14]. Protons, by a better sparing of surrounding healthy tissue, may be able to increase the dose to the tumour without an increase in toxicity [15]. If the present study demonstrates a decrease in toxicity for equal-dose radiotherapy allied with protons versus photons, increasing radiation doses with protons within a clinical trial would be the next step to achieve better local tumour control, which may then also be related to increased overall survival and metastasis-free survival as shown by others [16].

\section{Trial status}

The trial is currently recruiting.

\section{Additional files}

Additional file 1: Table S1. SPIRIT 2013 checklist PRONTOX. (DOC $121 \mathrm{~kb}$ ) Additional file 2: Figure S1. SPIRIT 2013 figure PRONTOX. (DOC 51 kb)

\section{Abbreviations}

3D-CRT: three-dimensional conformal radiation therapy; 4D-CT: Four-dimensional computer tomography; CT: Computer tomography; CTC-AE: Common Terminology Criteria for Adverse Events; CTV: Clinical target volume; FDG: Fluorodeoxyglucose; GTV: Gross tumour volume; Gy: Gray; iGTV: Internal gross tumour volume; IMRT: Intensity-modulated radiation therapy; MRI: Magnetic resonance imaging; NSCL: Non-small-cell lung carcinoma; OAR: Organs at risk; PET: Positron Emission Tomography; RBE: Relative biological effectiveness 


\section{Acknowledgements}

Julia Thiele, Annika Jakobi and Dr. Cordelia Hoinkis are acknowledged for their efforts in quality assurance of both treatment modalities being assessed in this phase II clinical trial.

\section{Funding}

The study will be funded by institutional funding within the German cancer consortium (DKTK).

\section{Availability of data and materials}

Not applicable.

\section{Authors' contributions}

SZ drafted the trial protocol and the manuscript. SL calculated the underlying statistics. MS helped to draft the trial protocol and the manuscript. ET, SA, CR, RB and PW contributed to drafting the protocol and implementing the study in clinical practice. MK, ET and MB provided trial ideas, and reviewed the trial protocol and manuscript. SM calculated IMRT and proton plans for comparison. $\mathrm{KS}$ and $\mathrm{CR}$ reviewed the protocol with respect to feasibility of clinical implementation. All authors read and approved the manuscript.

\section{Authors' information}

All authors are members of the protocol committee and read and approved the trial protocol and the final manuscript.

\section{Competing interests}

The authors declare that they have no competing interests.

\section{Consent for publication}

There are no restrictions concerning publications by any funding source.

\section{Ethics approval and consent to participate}

Approval to conduct this study (version 2.0 from 23 April 2015) was granted by the University Ethics Committee of the Dresden Medical Faculty (EK12012015). Informed consent of patients is mandatory before recruitment.

\section{Author details}

'Department of Radiation Oncology, Faculty of Medicine and University Hospital Carl Gustav Carus, Technische Universität Dresden, OncoRay National Center for Radiation Research in Oncology, Faculty of Medicine and University Hospital Carl Gustav Carus, Technische Universität Dresden and Helmholtz-Zentrum Dresden - Rossendorf, German Cancer Consortium (DKTK) Dresden and German Cancer Research Center (DKFZ), National Center for Tumor Diseases (NCT), Partner Site Dresden, Fetscherstr. 74, Dresden 01307, Germany. ${ }^{2}$ German Cancer Consortium (DKTK), German Cancer Research Center (DKFZ), Department of Radiation Oncology, Faculty of Medicine and University Hospital Carl Gustav Carus, Technische Universität Dresden, National Center for Tumor Diseases (NCT), Partner Site Dresden, Fetscherstr. 74, Dresden 01307, Germany. ${ }^{3}$ OncoRay - National Center for Radiation Research in Oncology, Faculty of Medicine and University Hospital Carl Gustav Carus, Technische Universität Dresden and Helmholtz-Zentrum Dresden - Rossendorf, National Center for Tumor Diseases (NCT), Partner Site Dresden, Fetscherstr. 74, Dresden 01307, Germany. ${ }^{4}$ Helmholtz-Zentrum Dresden - Rossendorf, Institute of Radiooncology, Department of Radiation Oncology, Faculty of Medicine and University Hospital Carl Gustav Carus, Technische Universität Dresden, OncoRay - National Center for Radiation Research in Oncology, German Cancer Consortium (DKTK), German Cancer Research Center (DKFZ), National Center for Tumor Diseases (NCT), Partner Site Dresden, Bautzner Landstr. 400, Dresden 01328, Germany. ${ }^{5}$ OncoRay National Center for Radiation Research in Oncology, Faculty of Medicine and University Hospital Carl Gustav Carus, Technische Universität Dresden, Helmholtz-Zentrum Dresden - Rossendorf, Fetscherstr. 74, PF 41, Dresden 01307, Germany. ${ }^{6}$ Department of Radiation Oncology, Faculty of Medicine and University Hospital Carl Gustav Carus, Technische Universität Dresden, National Center for Tumor Diseases (NCT), Partner Site Dresden, Fetscherstr. 74, Dresden 01307, Germany. ${ }^{7}$ Department of Radiation Oncology, Faculty of Medicine and University Hospital Carl Gustav Carus, Technische Universität Dresden, OncoRay - National Center for Radiation Research in Oncology, Faculty of Medicine and University Hospital Carl Gustav Carus, Technische Universität Dresden and Helmholtz-Zentrum Dresden - Rossendorf, National Center for Tumor Diseases (NCT), Partner Site Dresden, Fetscherstr. 74,
Dresden 01307, Germany. ${ }^{8}$ OncoRay - National Center for Radiation Research in Oncology, Faculty of Medicine and University Hospital Carl Gustav Carus and Helmholtz-Zentrum Dresden - Rossendorf, Technische Universität Dresden,German Cancer Consortium (DKTK) Dresden and German Cancer Research Center (DKFZ), Heidelberg, Helmholtz-Zentrum Dresden Rossendorf, Institute of Radiooncology, Fetscherstr. 74, Dresden 01307, Germany. ${ }^{9}$ Department of Radiation Oncology, Faculty of Medicine and University Hospital Carl Gustav Carus, Technische Universität Dresden, OncoRay - National Center for Radiation Research in Oncology, Faculty of Medicine and University Hospital Carl Gustav Carus, Technische Universität Dresden and Helmholtz-Zentrum Dresden - Rossendorf, German Cancer Consortium (DKTK) Dresden and German Cancer Research Center (DKFZ) Heidelberg, Helmholtz-Zentrum Dresden - Rossendorf, Institute of Radiooncology, National Center for Tumor Diseases (NCT), Partner Site Dresden, Fetscherstr. 74, Dresden 01307, Germany. ${ }^{10}$ Department of Radiation Oncology, Faculty of Medicine and University Hospital Carl Gustav Carus, Technische Universität Dresden, OncoRay - National Center for Radiation Research in Oncology, Faculty of Medicine and University Hospital Carl Gustav Carus, Technische Universität Dresden and Helmholtz-Zentrum Dresden - Rossendorf German Cancer Consortium (DKTK) Dresden, German Cancer Research Center (DKFZ) Heidelberg, Helmholtz-Zentrum Dresden Rossendorf, National Center for Tumor Diseases (NCT), Partner Site Dresden, Fetscherstr. 74, Dresden 01307, Germany.

Received: 26 May 2016 Accepted: 3 November 2016 Published online: 15 November 2016

\section{References}

1. Aupérin A, Le Péchoux C, Pignon JP, Koning C, Jeremic B, Clamon G, et al. Concomitant radio-chemotherapy based on platin compounds in patients with locally advanced non-small cell lung cancer (NSCLC): a meta-analysis of individual data from 1764 patients. Ann Oncol. 2006;17(3):473-83.

2. Bradley JD, Paulus R, Komaki R, Masters G, Blumenschein G, Schild S, et al. Standard-dose versus high-dose conformal radiotherapy with concurrent and consolidation carboplatin plus paclitaxel with or without cetuximab for patients with stage IIIA or IIIB non-small-cell lung cancer (RTOG 0617): a randomised, two-by-two factorial phase 3 study. Lancet Oncol. 2015;16(2):187-99.

3. Nalbantov G, Kietselaer B, Vandecasteele K, Oberije C, Berbee M, Troost E, Dingemans A-M, et al. Cardiac comorbidity is an independent risk factor for radiation-induced lung toxicity in lung cancer patients. Radiother Oncol. 2013;109(1):100-6.

4. Sejpal S, Komaki R, Tsao A, Chang JY, Liao Z, Wei X, et al. Early findings on toxicity of proton beam therapy with concurrent chemotherapy for nonsmall cell lung cancer. Cancer. 2011;117(13):3004-13.

5. Vogelius IR, Westerly DC, Aznar MC, Cannon GM, Korreman SS, Mackie TR, et al. Estimated radiation pneumonitis risk after photon versus proton therapy alone or combined with chemotherapy for lung cancer. Acta Oncol. 2011;50(6):772-6.

6. Palma DA, Senan S, Oberije C, Belderbos J, de Dios NR, Bradley JD, et al. Predicting esophagitis after chemoradiation therapy for non-small cell lung cancer: an individual patient data meta-analysis. Int J Radiat Oncol. 2013;87(4):690-6.

7. Palma DA, Senan S, Tsujino K, Barriger RB, Rengan R, Moreno M, et al. Predicting radiation pneumonitis after chemoradiation therapy for lung cancer: an international individual patient data meta-analysis. Int J Radiat Oncol. 2013;85(2):444-50.

8. Makimoto T, Tsuchiya S, Hayakawa K, Saitoh R, Mori M. Risk factors for severe radiation pneumonitis in lung cancer. Jpn J Clin Oncol. 1999;29(4):192-7.

9. Marcu LG. Altered fractionation in radiotherapy: from radiobiological rationale to therapeutic gain. Cancer Treat Rev. 2010;36(8):606-14.

10. Kollar L, Rengan R. Stereotactic body radiotherapy. Semin Oncol. 2014;41(6): 776-89.

11. Choi N, Baumann M, Flentjie M, Kellokumpu-Lehtinen P, Senan S, Zamboglou N, et al. Predictive factors in radiotherapy for non-small cell lung cancer: present status. Lung Cancer. 2001;31(1):43-56.

12. van Baardwijk A, Wanders S, Boersma L, Borger J, Ollers M, Dingemans A-M, Bootsma $\mathrm{G}$, et al. Mature results of an individualized radiation dose prescription study based on normal tissue constraints in stages I to ||| non-small-cell lung cancer. J Clin Oncol. 2010;28(8):1380-6. 
13. Hoffmann A, Troost E, Huizenga H, Kaanders J, Bussink J. Individualized dose prescription for hypofractionation in advanced non-small-cell lung cancer radiotherapy: an in silico trial. Int J Radiat Oncol. 2012;83(5):1596-602.

14. Baumann M, Herrmann T, Koch R, Matthiessen W, Appold S, Wahlers B, et al. Final results of the randomized phase III CHARTWEL-trial (ARO 97-1) comparing hyperfractionated-accelerated versus conventionally fractionated radiotherapy in non-small cell lung cancer (NSCLC). Radiother Oncol. 2011;100(1):76-85.

15. Hoppe BS, Flampouri S, Henderson RH, Pham D, Bajwa AA, D'Agostino H, et al. Proton therapy with concurrent chemotherapy for non-small-cell lung cancer: technique and early results. Clin Lung Cancer. 2012;13(5):352-8.

16. Saunders M, Dische S, Barrett A, Harvey A, Griffiths G, Palmar M. Continuous, hyperfractionated, accelerated radiotherapy (CHART) versus conventional radiotherapy in non-small cell lung cancer: mature data from the randomised multicentre trial. CHART Steering Committee. Radiother Oncol. 1999;52(2):137-48

Submit your next manuscript to BioMed Central and we will help you at every step:

- We accept pre-submission inquiries

- Our selector tool helps you to find the most relevant journal

- We provide round the clock customer support

- Convenient online submission

- Thorough peer review

- Inclusion in PubMed and all major indexing services

- Maximum visibility for your research

Submit your manuscript at www.biomedcentral.com/submit
Biomed Central 